\title{
The Epigenetic Influence of Tumor and Embryonic Microenvironments: How Different are They?
}

\author{
Daniel E. Abbott • Caleb M. Bailey • \\ Lynne-Marie Postovit • Elisabeth A. Seftor • \\ Naira Margaryan • Richard E. B. Seftor • \\ Mary J. C. Hendrix
}

Received: 19 December 2007 / Accepted: 22 January 2008/Published online: 20 February 2008

(C) Springer Science + Business Media B.V. 2008

\begin{abstract}
The microenvironment is being increasingly recognized as a critical component in tumor progression and metastases. As such, the bi-directional signaling of extracellular mediators that promote tumor growth within the microenvironment is a focus of intense scrutiny. Interestingly, there are striking similarities between the phenotypes of aggressive tumor and embryonic stem cells, particularly with respect to specific signaling pathways underlying their intriguing plasticity. Here, we demonstrate the epigenetic influence of the hESC microenvironment on the reprogramming of aggressive melanoma cells using an innovative 3-D model. Specifically, our laboratory has previously demonstrated the redifferentiation of these melanoma cells to a more melanocyte-like phenotype (Postovit et al., Stem Cells 24(3):501-505, 2006), and now we show the loss of VE-Cadherin expression (indic-
\end{abstract}

Daniel E. Abbott, Caleb M. Bailey, Lynne-Marie Postovit, Elisabeth A. Seftor contributed equally.

\section{E. Abbott}

Children's Memorial Research Center, Department of Surgery,

Northwestern University/Feinberg School of Medicine,

Chicago, IL 60614, USA

C. M. Bailey $\cdot$ E. A. Seftor $\cdot$ N. Margaryan

Cancer Biology and Epigenomics Program,

Children's Memorial Research Center,

Chicago, IL 60614, USA

L.-M. Postovit

Department of Anatomy and Cell Biology,

Schulich School of Medicine and Dentistry,

University of Western Ontario,

Medical Sciences Building, Rm. 438,

London, ON N6A 5C1, Canada ative of a plastic vasculogenic phenotype) and the loss of Nodal expression (a plasticity stem cell marker) in tumor cells exposed to the hESC microenvironment. Further studies with the 3-D culture model revealed the epigenetic influence of aggressive melanoma cells on hESCs resulting in the down-regulation of plasticity markers and the emergence of phenotype-specific genes. Additional studies with the aggressive melanoma conditioned matrix microenvironment demonstrated the transdifferentiation of normal melanocytes into melanoma-like cells exhibiting a vasculogenic phenotype. Collectively, these studies have advanced our understanding of the epigenetic influence associated with the microenvironments of hESCs and aggressive melanoma cells, and shed new light on their therapeutic implications. Moreover, we have a better appreciation of the convergence of embryonic and tumorigenic signaling path-

\author{
R. E. B. Seftor • M. J. C. Hendrix ( $\square)$ \\ Cancer Biology and Epigenomics Program, \\ Children's Memorial Research Center, \\ Robert H. Lurie Comprehensive Cancer Center, \\ Northwestern University/Feinberg School of Medicine, \\ 2430 North Halsted Street, P.O. Box 222, \\ Chicago, IL 60614, USA \\ e-mail: mjchendrix@childrensmemorial.org
}


Table 1 A comparison of select embryonic markers expressed in human embryonic stem cells vs. aggressive melanoma cells

Gene expression

Gene name Human embryonic stem cells (H9)

Aggressive melanoma cells (C8161)

\begin{tabular}{lll}
\hline Nodal & +++ & ++ \\
Lefty & +++ & - \\
Cripto & +++ & $+/-$ \\
Oct3/4 & ++ & - \\
Nanog & ++ & -
\end{tabular}

Relative gene expression by real time PCR analysis of select cell types designated by: +++ significant expression; ++ moderate expression; + expression by a subpopulation of cells; and - no expression.

ways that might stimulate further consideration of targeting Nodal in aggressive tumor cells resulting in a downregulation of tumorigenic potential and plasticity.

Keywords Embryonic stem cells · Microenvironment . Epigenetic $\cdot$ Nodal $\cdot$ Melanoma

\section{Introduction}

Recent attention has been given to comparisons between stem cells and aggressive cancer cells. Indeed, similarities such as unlimited proliferative potential, cellular plasticity, and the expression of genes involved in maintaining pluripotency and/or plasticity are shared by these two different cell types (Table 1) [2, 3]. Specifically, recent studies have revealed a convergence of critical pathways associated with both cancer progression and normal embryologic development including Hedgehog, Wingless (Wnt), Notch and Nodal signaling pathways [4-7]. The identification of these shared pathways has led to a more comprehensive examination of the bi-directional microenvironmental communication propagated among cells through at least three different mechanisms: direct cell-cell contact, autocrine and paracrine signaling driven by soluble secreted factors, and modeling (or re-modeling) of the extracellular matrix. Each mechanism plays an important role in both normal developmental pathways and malignant cancer development and progression.

The microenvironment can exert profound epigenetic effects on cells through interactions between cells, or through cell-derived factors deposited into the microenvironment. These effects can be transient, as seen with signaling pathways regulating cellular proliferation and migration, or associated with more stable events, such as cell fate determination and differentiation. While normal development in the embryo is coordinated by a complex equilibrium between positively and negatively acting cues and signals, our work and that of others have demonstrated an aberrant expression of potent, embryonic morphogens by some aggressive cancer cell types which, in the absence of their normal negative regulators, induce uncontrolled proliferation, increased survival, dedifferentiation and plasticity in these specific cancer cells $[5,8,9]$. This combination of increased survival and plasticity promotes malignant transformation and contributes to metastasis.

Given the critical role of the microenvironment in cellular regulation and the observation that gene expression profiles and certain phenotypic characteristics are shared by human embryonic stem cells (hESCs) and some aggressive melanoma cells, we examined potential similarities and differences between the epigenetic influence of microenvironments associated with hESCs and aggressive melanoma cells. Since the embryonic microenvironment possesses key regulatory cues and signaling molecules that function to maintain and regulate the growth of the stem cell population, we hypothesized that an embryonic microenvironment might be able to influence cancer cells by normalizing their plastic phenotype. The concept that an embryonic microenvironment could reprogram cancer cells was elegantly highlighted by studies in the early 1970 s by Mintz and Illmensee who reported that teratocarcinoma cells injected into murine blastocysts demonstrated totipotency and a reversion to a normal phenotype [10, 11]. This model firmly established a non-mutational basis for the transformation of these teratocarcinoma cells to a malignant phenotype, and demonstrated the unique ability of an embryonic microenvironment to reprogram these cells to a normal phenotype. These studies were corroborated in an avian model during the 1980s by Dolberg and Bissell who showed that Rous sarcoma virus injected in the chick embryo would not induce sarcoma formation [12]. Our laboratory and others recently extended these studies and demonstrated that the chick and zebrafish embryonic microenvironments (approximately six to eight somite stage in chick development and blastula stage in zebrafish) not only repress tumor formation when implanted with aggressive human melanoma cells, but induce lineage-specific genes and migratory behaviors associated with a more normal phenotype - representative of an epigenetic reprogramming event $[13,14]$. Additional studies by Haldi and coworkers demonstrated that metastatic melanoma cells implanted in the zebrafish microenvironment 2 days postfertilization retained their enhanced proliferative, migratory and tumorigenic characteristics [15]. These observations leant further support to the concept that the early-stage embryonic microenvironment is unique and specific to events associated with the ability to reprogram aggressive melanoma cells.

While these seminal studies demonstrated that the embryonic microenvironment could have significant influ- 
ence on the phenotypic characteristics of aggressive cancer cells, the converse - an evaluation of the effects of a tumorigenic microenvironment on embryonic stem cells-has not been sufficiently explored. Recent studies by Karnoub and colleagues have revealed key signaling pathways between bone marrow-derived mesenchymal stem cells and human breast cancer cells, and sought to identify the relationship between the tumorigenic microenvironment and tissuespecific stem cell populations [16]. In light of the observation that there are unique phenotypic characteristics shared by both embryonic stem cells and aggressive melanoma cells which may be profoundly influenced by the embryonic microenvironment, we examined the ability of their respective microenvironments to affect and/or influence the phenotype of the other. In this review, we present an evaluation of recent data illuminating the effects of the hESC-conditioned matrix microenvironment on aggressive C8161 melanoma cells as well as novel and dramatic findings concerning the effects of the C8161 melanoma-conditioned matrix microenvironment on hESCs. These observations may lead to the development of novel cancer therapeutics based on targeting converging pathways leading to a reversal of the dedifferentiated, plastic tumor cell phenotype.

\section{Embryonic Microenvironment}

The embryonic microenvironment is a dynamic yet finely regulated network of cellular communication that is critical to cell behavior and fate determination throughout embryogenesis and beyond. Molecular cues in the embryonic milieu, originating from a variety of cell types, possess various signaling characteristics with diverse effects on specific target cells or tissues. Certain stimulatory factors such as Activin, Nodal and FGF maintain pluripotency in embryonic stem cells, while other factors, such as ngn3, Notch, and ASK-1 (endocrine, hematopoietic, and neuronal lineages, respectively), dictate a differentiated fate [17-20]. These signals regulate the phenotype of stem cells or their progeny and are at times antagonistic, and may be separated in a spatial and/or temporal fashion. These processes are exquisitely complex, and their sophistication continues to be appreciated as molecular profiling of embryonic stem cells and their microenvironment(s) is continually studied and analyzed [21-23]. However, despite an incomplete understanding of the full scope of microenvironmental contributions to organism development, health, and disease, exciting insights into the influence of embryonic stem cells and their regulatory properties have been made. These breakthroughs in embryonic stem cell biology offer tremendous promise in numerous therapeutic arenas, most notably regenerative technologies and oncologic therapy. It is through further investigation into this genre that such breakthroughs will be realized.
The complexity of the embryonic microenvironment is determined by a number of factors. Perhaps most important is the cellular composition which dictates the temporal and spatial distribution of signaling molecules which then direct cell fate determination, embryogenesis and appropriate apoptotic cues [24, 25]. These factors orchestrate elegant developmental steps in a regulated fashion, with the inhibitory signaling molecules among the most important factors within the embryonic milieu, as they regulate appropriate growth and differentiation while limiting unabated development [26-28]. For example, Lefty is a stem-cell derived inhibitor of the Activin-Nodal signaling pathway that promotes differentiation of stem cells and may play an important role in normal gastrulation and neural tube formation [29-31]. This regulation is critical and will be discussed later, specifically with respect to tumorigenesis.

Based on the concept that the hESC microenvironment provides a dynamic regulatory niche for stem cell function, we explored the possibility that this embryonic microenvironment could reprogram the plastic tumor cell phenotype. To address the need for a novel experimental design that expands on standard cellular gene and protein expression analysis, our laboratory has developed an in vitro model that employs a 3-D matrix (Fig. 1) to assess microenvironmental properties and influences when conditioned by specific cells types, primarily hESCs (aggressive cancer cells may also be used-to be discussed in more detail later). Briefly, cells are plated on a 3-D matrix for 3-4 days followed by lysis and removal using a dilute concentration

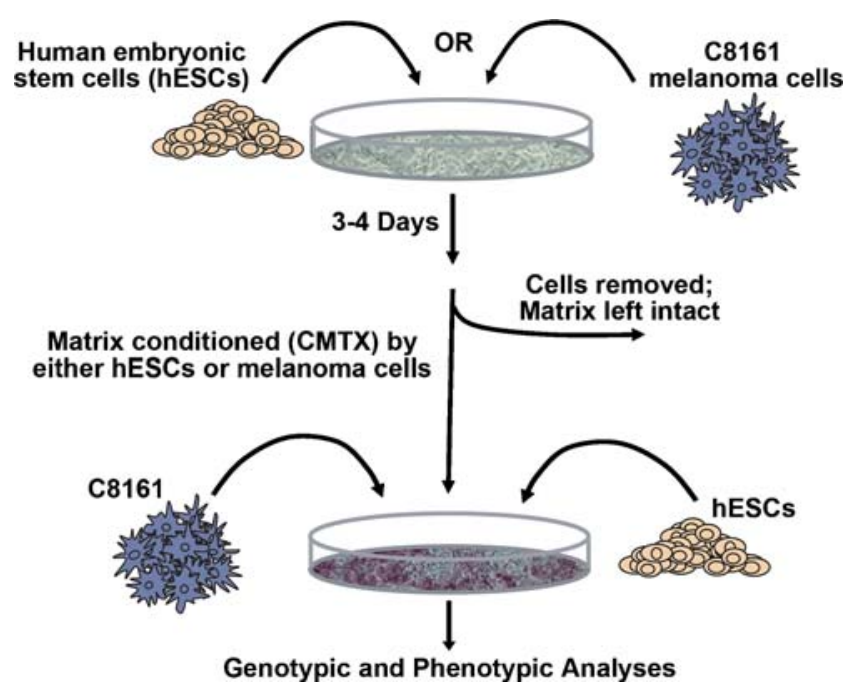

Fig. 13 -D model to study the epigenetic influence of the microenvironment on cell phenotype. Compact colonies of human embryonic stem cells (hESCs) or cancer cells are seeded onto a 3-D matrix (composed of Matrigel or type I collagen) for 3 to 4 days, then removed, resulting in a conditioned 3-D matrix (CMTX) onto which other cell types (such as hESCs, cancer cells, or normal melanocytes) are seeded and incubated for 3-4 days. Subsequently, changes in cell morphology, gene and protein expression as well as behavioral function(s) of the cells can be examined 
of ammonium hydroxide. Cells of interest are subsequently re-plated on this conditioned matrix (CMTX). Following 3-4 days of growth on the conditioned matrix, cells are removed for morphological, proliferative, functional, RNA or protein analysis. This approach permits a comprehensive analysis of the myriad influences a conditioned microenvironment may exert on neighboring cells, and provides a foundation from which further work may be designed and undertaken.

Both hESCs and certain aggressive cancer cells have the ability to affect/alter their respective microenvironments to induce epigenetic effects on subsequent cell populations when placed into these conditioned microenvironments. In particular, an extracellular matrix conditioned by hESCs results in dramatic changes in cells introduced into this environment. Figure 2a reveals the profound morphological

a

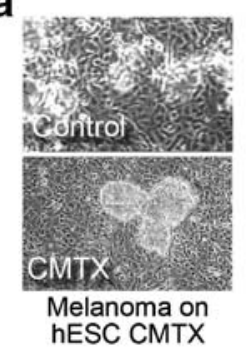

b
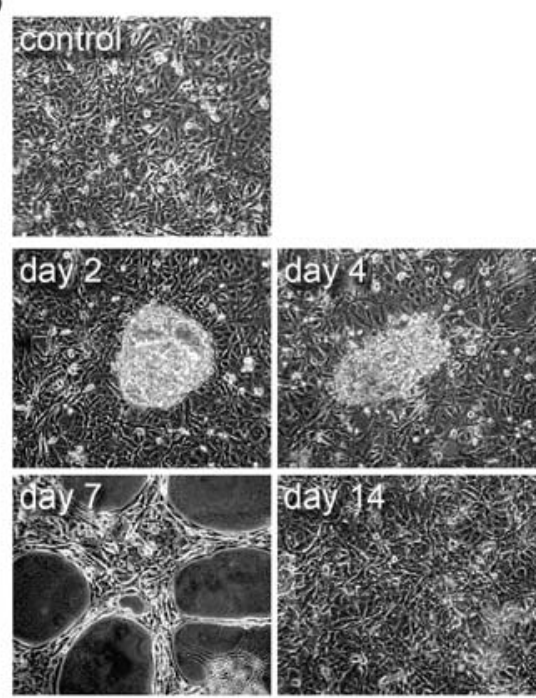

C

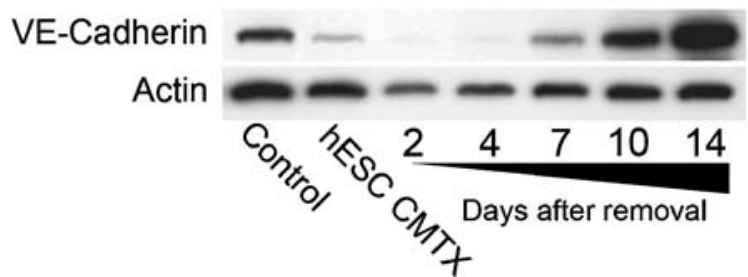

Fig. 2 The microenvironment of hESCs epigenetically inhibits the vasculogenic phenotype of aggressive melanoma cells. a Phasecontrast microscopy of C8161 metastatic melanoma cells grown on a control Matrigel matrix (upper panel) and induced to form spheroids (a marked morphological alteration resembling hESC colonies) following exposure to a 3-D hESC microenvironment ( $h E S C$ CMTX; lower panel). $\mathbf{b}$ This induction of spheroid formation is reversible as C8161 melanoma cells exposed to control Matrigel matrix following exposure to hESC CMTX are able to resume their plastic phenotype and form vasculogenic-like networks following a 7-day recovery period. $\mathbf{c}$ Western blot analysis of VE-Cadherin protein in C8161 cells exposed for 3 days to either control (unconditioned) Matrigel or to Matrigel conditioned by hESCs ( $E E S C$ CMTX). Some cancer cells exposed to the hESC CMTX were subsequently recovered on control (unconditioned) Matrigel for 2 to 14 days prior to Western blot analysis. Actin is used as a loading control changes that aggressive melanoma cells (C8161) display following culture on an hESC CMTX. Compared to controls (C8161 cells grown on an unconditioned control matrix; top panel), where melanoma cells grow primarily in an over-confluent monolayer, these same cells form spheroidal clusters in a manner similar to the morphology characteristic of stem cells cultured on an hESC CMTX (bottom panel).

The epigenetic nature of this transformation is especially evident after the melanoma cells are removed from the hESC CMTX and cultured under normal (non-embryonic) conditions. After removal from the influence of the hESC CMTX, the melanoma cells revert to their original morphologic appearance by 7 days. Figure 2 b depicts a 2 -week time-course during which C8161 cells are cultured on an hESC CMTX for 3 days and removed. Each panel shows the morphological changes associated with reversion back to an aggressive phenotype as more time elapses postexposure to an hESC CMTX. At 2 days, just as in Fig. 2a (lower panel), aggressive melanoma cells appear as spheroidal clusters, reminiscent of hESC colonies. By day 4 (panel b; post-removal from an hESC CMTX and re-plating on a control matrix), this spheroid morphology begins to diminish while at day 7 , the vasculogenic phenotype associated with aggressive melanoma cells is quite evident and any similarity to the embryoid body morphology of an ES cell cluster has diminished significantly. Two weeks following removal from an hESC CMTX, the aggressive C8161 cells have lost all semblance of an hESC cluster and appear identical to control melanoma cells that had never been exposed to an hESC CMTX. Such dramatic phenotypic changes demonstrate the profound influence of an embryonic conditioned microenvironment on aggressive tumor cells - during the time of exposure - and also highlight the transient nature of the epigenetic event.

Coincident with this morphologic transformation is an alteration in protein expression which is also induced by the hESC CMTX. For example, VE-Cadherin (an endothelial cell-associated gene product associated with vasculogenic mimicry in malignancy) is expressed in abundance by aggressive melanoma cells $[32,33]$. When aggressive melanoma cells are exposed to an hESC CMTX, VECadherin expression is significantly down-regulated with the greatest response seen at 4 days after removal of melanoma cells from the conditioned microenvironment (Fig. 2c). By day 14 post-removal from the hESC CMTX, the cells express levels of VE-Cadherin equivalent to the control (untreated) cells within the same period of time concurrent with their morphological reversion to their original phenotype. These data corroborate past findings in our laboratory which showed that melanoma cells exposed to an hESC CMTX underwent a $32 \%$ increase in methylation within a putative transcription factor binding 
site of the Nodal gene, as well as a substantial increase in melan-A production, exhibiting a more melanocytic (benign) phenotype $[1,34]$. This demonstration of epigenetic modulation by an embryonic microenvironment illustrates the extraordinary influence that the microenvironment can have on cellular architecture, behavior and gene expression. These findings underscore the possibility of defining factor (s) within the hESC microenvironment that might downregulate the plasticity of aggressive melanoma cells, as demonstrated in Fig. 2.

\section{Cancer Microenvironment}

An impressive body of work has been performed in assessing the effects of cancer cells and their influence on the microenvironment. Through specific signaling molecules and re-modeling of their extracellular milieu (both cellular and stromal), aggressive cancer cells (or perhaps particular cancer stem cells) have the ability to enhance their tumorigenicity in functioning as a dynamic entity within their surroundings [35]. Our laboratory, and others, have highlighted key features of the extracellular space that play such a critical role in tumor remodeling, including laminin- $5 \gamma 2$, matrix metalloproteinases and other basement membrane constituents $[36,37]$. The importance of the tumor microenvironment has been realized from a therapeutic perspective as well, in that a number of treatments that target the extracellular components of tumors are in development or clinical trials [38, 39].

An example of how aggressive cancer cells (with an embryonic-like phenotype) may also affect their microenvironment to induce phenotypic changes in other cells is illustrated by culturing normal human melanocytes (which exhibit a homogeneously benign phenotype) on a matrix conditioned by aggressive melanoma cells (C8161). Figure 3 shows that C8161 cells cultured on a 3-D type I collagen matrix produce visible networks (delineated by arrows; left panel) as part of their vasculogenic mimicry phenotype. After the aggressive cancer cells were removed, human melanocytes were placed on the conditioned matrix, inducing these normal cells to form vasculogenic mimicry-like networks. Coincident with the initiation of the more aggressive phenotype was an induction of VE-Cadherin expression, suggesting an epigenetic modification consistent with a vascular cell-like molecular profile, similar to that of the aggressive melanoma phenotype [40]. This result highlights the powerful influence that certain cell types have on their microenvironment and in this instance, the bidirectional communication between normal melanocytes and a cancerous microenvironment led to the acquisition of tumor cell-like characteristics.

Melanocytes are not the only cell type susceptible to this kind of epigenetic modulation by a cancerous microenvironment. When hESCs are cultured on a 3-D matrix conditioned by aggressive melanoma cells for 3 days, their morphologic transformation is striking (Fig. 4a). Rather than typical spheroidal clusters under control conditions (top left panel), hESCs begin to flatten on the matrix, migrate as a monolayer, and exhibit a mesenchymal-like morphology — characteristics associated with the aggressive melanoma cells that conditioned this microenvironment. This dramatic change in cell morphology is associated with intriguing gene expression changes as well (Fig. 4b). hESCs cultured on an C8161 CMTX for 3 days and harvested for mRNA analysis demonstrate: (1) a significant

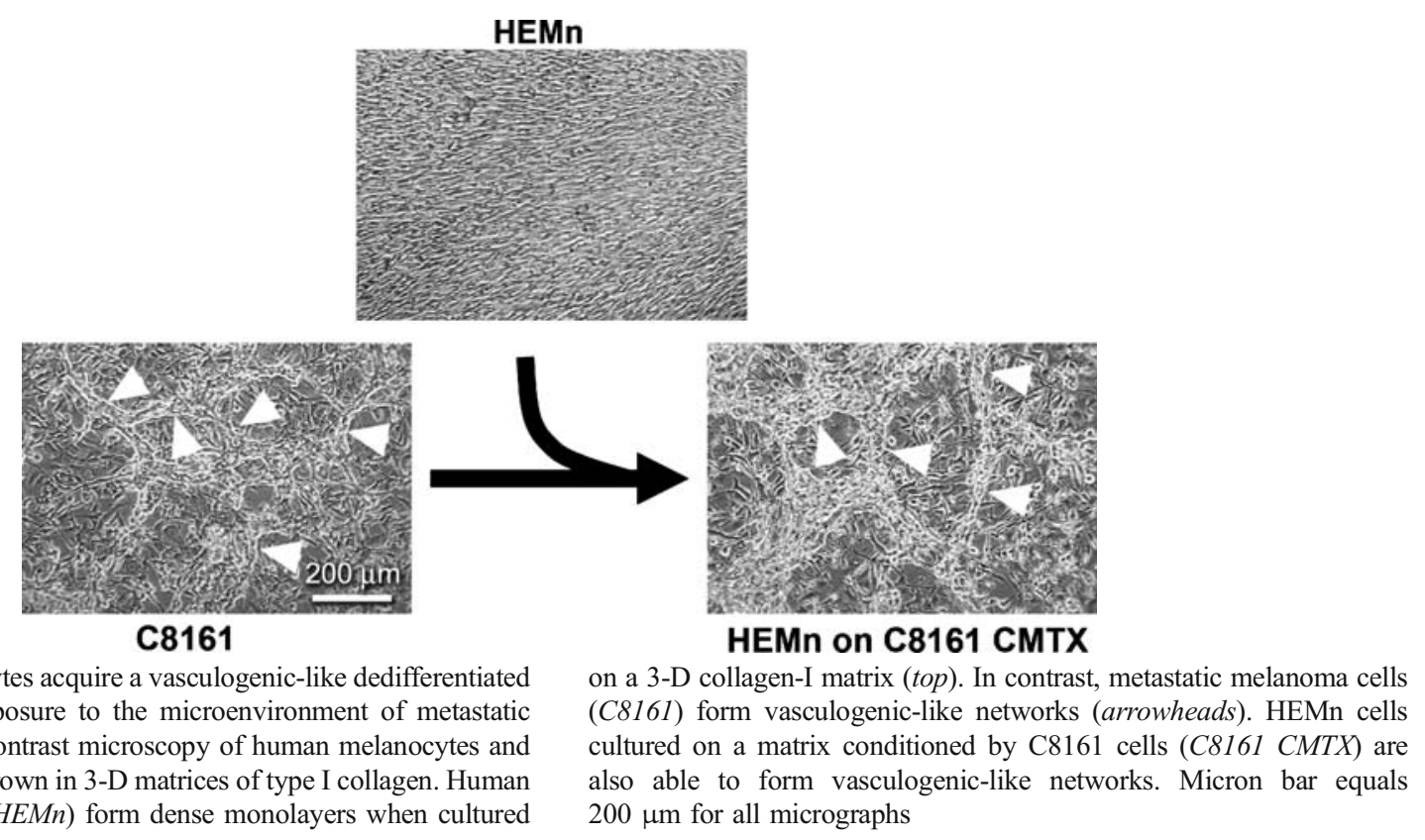

Fig. 3 Normal melanocytes acquire a vasculogenic-like dedifferentiated phenotype following exposure to the microenvironment of metastatic melanoma cells. Phase-contrast microscopy of human melanocytes and C8161 melanoma cells grown in 3-D matrices of type I collagen. Human epidermal melanocytes (HEMn) form dense monolayers when cultured 
a
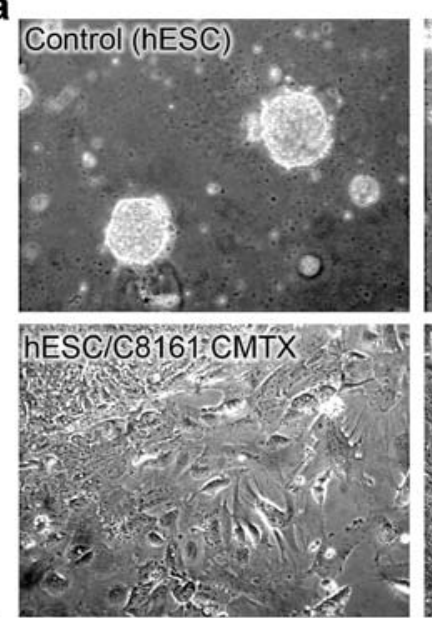

b

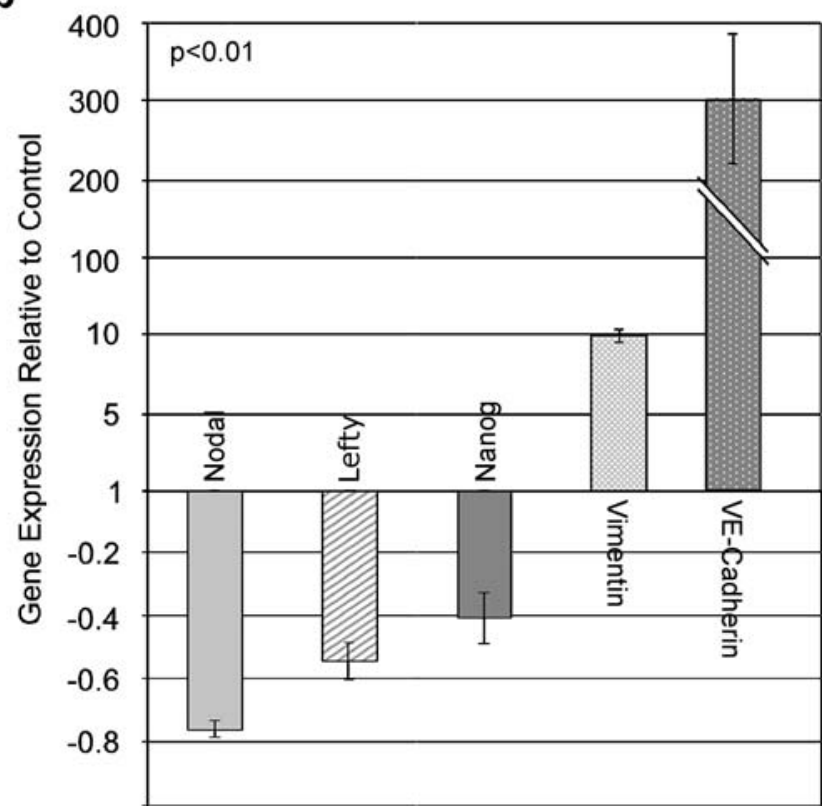

Fig. 4 hESCs exhibit a vasculogenic and mesenchymal-like phenotype following exposure to the microenvironment of metastatic melanoma cells. a Phase-contrast microscopy of hESCs grown on 3D Matrigel matrices. Under control conditions, hESCs grow as densely packed undifferentiated colonies. In contrast, hESCs cultured on a matrix conditioned by C8161 cells (C8161 CMTX) exhibit an altered phenotype reminiscent of cell differentiation. b Real time RTPCR analysis of Nodal, Lefty, Nanog, Vimentin, and VE-Cadherin mRNA in hESCs exposed for 3 days to either control (unconditioned) Matrigel or to Matrigel conditioned by human metastatic melanoma cells (C8161 CMTX). Gene levels were normalized using RPLPO and bars represent mean gene expression \pm standard deviation normalized to gene expression in hESCs cultured under control conditions

down-regulation of markers associated with embryonic stem cell pluripotency and an undifferentiated state (Nodal, Lefty and Nanog), and (2) dramatic amplification of genes associated with a differentiated cellular phenotype (Vimentin and VE-Cadherin). Specifically, Nodal is suppressed to nearly $20 \%$ of control levels, consistent with the morphologic changes noted in Fig. 4a following exposure to an
C8161 CMTX. Lefty, one of Nodal's inhibitors and expressed primarily by undifferentiated hESCs, is found at less than $50 \%$ of control levels. Again, this finding is consistent with the notion that hESCs, induced by the cancerous microenvironment, lose this regulatory factor inherent to hESCs - and critical to cell fate determination. Furthermore, Nanog, a marker widely associated with 'stemness', is decreased in expression following hESC exposure to a melanoma tumor-conditioned microenvironment.

Conversely, when hESCs are exposed to the C8161 CMTX, two cell phenotype-associated markers are considerably up-regulated compared to hESC controls. Vimentin, an intermediate filament protein expressed first by cells of mesodermal origin and a marker of a mesenchymal cell type, is amplified nearly tenfold. Furthermore, as is observed following melanocyte exposure to an aggressive melanoma CMTX, the endothelial-specific marker VE-Cadherin is upregulated - in this circumstance almost 300-fold - in hESCs exposed to a microenvironment conditioned by aggressive melanoma tumor cells.

Taken together, these compelling data confirm that just as hESCs have the ability to condition their microenvironment and epigenetically modulate their surroundings, aggressive melanoma cells can also radically alter and manipulate their milieu without requiring cell-cell contact or direct communication. The soluble factors associated with a conditioned microenvironment may be derived from a multitude of cell types and signal either pro- or antitumorigenic effects. Using an approach based on our understanding of how cells confer influential cues via their extracellular microenvironment, novel therapeutic approaches may be designed which specifically target signaling molecules in the tumor microenvironment associated with cancer progression.

\section{Convergence of Embryonic and Tumorigenic Signaling Pathways}

The embryonic microenvironment, as discussed above, is by necessity tightly regulated via a number of signaling pathways, and our research group has focused on the Nodal signaling axis. Nodal is an embryonic morphogen responsible for left-right patterning in the embryo, maintenance of pluripotency/self-renewal in human embryonic stem cells and appears to play a critical role in maintaining the undifferentiated state of cells [20, 41, 42]. While Nodal expression is lost as hESCs lose their undifferentiated state and undergo differentiation, it is re-expressed in a dysregulated fashion in aggressive melanoma and breast cancer cells [2, 43].

The mechanisms of Nodal signaling are complex. Nodal binds to the ALK 4/5/7 and ActIIRB receptor complex utilizing Cripto as a co-receptor (member of the EGF-CFC 
a

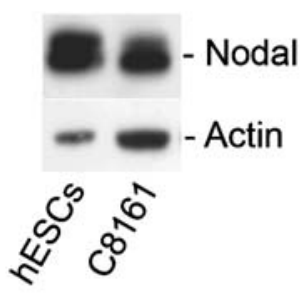

b

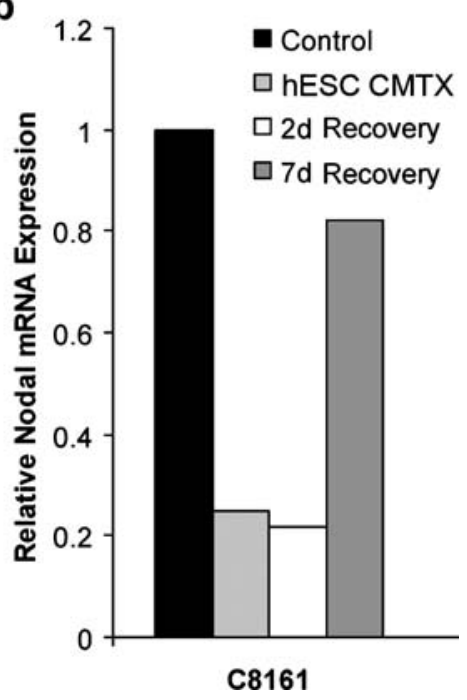

Fig. 5 Exposure to an hESC microenvironment decreases the expression of a potent embryonic-associated morphogen (Nodal) aberrantly expressed in aggressive melanoma cells. a Western blot analysis of Nodal in hESCs and in C8161 human metastatic melanoma cells. Actin is used as a loading control. b Real time RTPCR analysis of Nodal mRNA in C8161 melanoma cells exposed for 3 days to either fresh Matrigel (control) or to Matrigel conditioned by hESCs ( $h E S C s$ CMTX). Some cancer cells exposed to the CMTX were subsequently recovered on control (unconditioned) Matrigel for 2 or 7 days prior to analysis. Gene levels were normalized using $18 \mathrm{~s}$ and bars represent mean gene expression normalized to Matrigel control

family) to enhance its signal. This activation results in phosphorylation of ALK 4/5/7, which in turn phosphorylates the Smad 2/3 complex. This p-Smad 2/3 complex binds Smad 4 which in turn signals nuclear transcription of Nodal as well as Lefty, its natural inhibitor [44]. Each

ligand binding and signal transduction step also lends itself to possible therapeutic inhibition by small molecule inhibitors, immunoregulation and/or directed targeting of key components of the signaling pathway [43].

It is important to note that Nodal not only regulates itself via an autocrine, feed-forward mechanism [5], but it is also secreted where it exerts its effects locally through the microenvironment. The importance of secreted Nodal has been demonstrated in studies during which Nodal has been experimentally inhibited in a zebrafish embryo model. Nodal, when either ectopically injected or secreted by cancer cells transplanted into the embryo, induced secondary axis formation and ectopic cranial outgrowths in the zebrafish [5]. However, when Nodal is antagonized by either overexpression of Lefty- 1 in the zebrafish microenvironment $[45,46]$ or morpholino treatment $\left(\mathrm{Nodal}^{\mathrm{MO}}\right.$ in the cancer cells), ectopic axes and outgrowths are significantly reduced [5]. While these studies are compelling with respect to embryologic development, cell-fate determination and growth, the influence of Nodal secretion in cancer must still be elucidated.

Given the effects of Nodal and the Nodal signaling cascade in these model systems, we examined potential changes in Nodal expression in aggressive melanoma cells following exposure to an hESC CMTX. As shown by protein analysis in Fig. 5a, Nodal is highly expressed by both hESCs and aggressive melanoma cells (C8161). However, when melanoma cells are exposed to an hESC CMTX, Nodal mRNA expression is significantly downregulated by nearly $80 \%$ compared with control levels (Fig. 5b) and remains suppressed for several days after

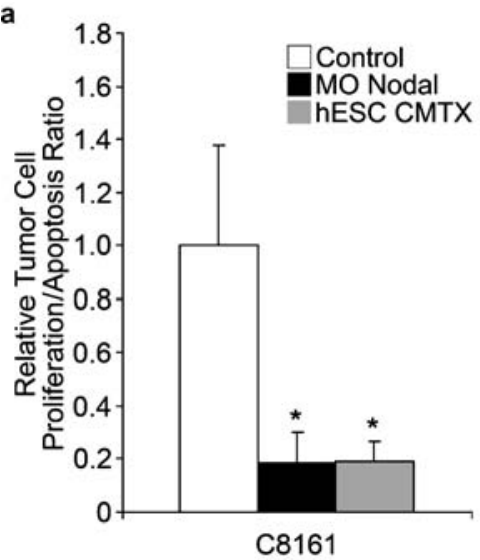

Fig. 6 Down-regulation of Nodal expression by exposure to the hESC microenvironment or by knock down with Nodal morpholino leads to abrogation metastatic melanoma tumorigenicity. a The ratio of tumor cell proliferation to apoptosis in tumors derived from human metastatic melanoma cells (C8161), determined by immunohistochemical staining for Ki-67 and terminal deoxynucleotidyl transferase biotin-dUTP nick-end labeling (TUNEL). Prior to injection into a mouse, C8161 cells were cultured for 3 days on control or hESC conditioned matrices ( $h E S C$ CMTX), or treated with MO Nodal to

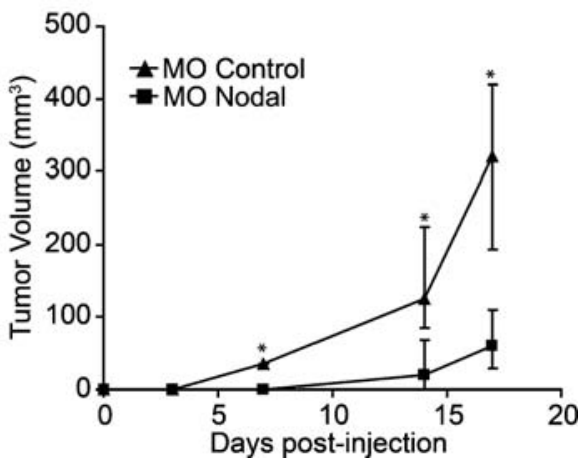

knock down Nodal expression. Bars represent mean normalized values \pm standard deviation, and values indicated by an asterisk are significantly different from control values $(p<0.05)$. b In vivo tumor formation in mice injected with Nodal morpholino-transfected C8161 cells (MO Nodal) vs. melanoma cells transfected with control morpholino ( $M O$ Control), introduced orthotopically subcutaneously. Values represent the median tumor volume $\left(\mathrm{mm}^{3}\right) \pm$ interquartile range $(n=5 /$ group,$p<0.05)$ 
the melanoma cells are removed from the hESC CMTX and grown on a control matrix. However, after 7 days of recovery the Nodal mRNA levels return to near control levels in the melanoma cells. This inhibition of Nodal by a microenvironment conditioned by hESCs suggests a potential therapeutic target based on an anti-Nodal treatment regime that merits further scientific investigation.

Since Nodal is expressed and secreted in a dysregulated fashion by aggressive melanoma cells which do not express Lefty (an endogenous Nodal inhibitor, as shown in Table 1), a direct assumption would be that targeted disruption of Nodal could have significant effects on tumor cell growth. This was shown to be the case when Nodal morpholino treatment (morpholine ring-based anti-sense nucleotides) of C8161 cells was found to decrease the proliferation/ apoptosis ratio of the cells by nearly $80 \%$ compared to controls (Fig. 6a). A similar effect was seen when the C8161 cells were cultured on an hESC CMTX, and might be attributed to the deposition of inhibitors of Nodal, such as Lefty, into the hESC CMTX. These in vitro data are supported by in vivo experiments which demonstrate and confirm the effects of Nodal inhibition on tumor growth [5]. C8161 melanoma cells treated with Nodal morpholino and harvested for xenograft transplantation in the subcutaneous tissue of nude mice exhibit significantly less tumor formation and growth than cells treated with control morpholinos (Fig. 6b). More than 2 weeks following implantation, tumor volume in treated C8161 xenografts remained less than $20 \%$ of controls. After 2 weeks, Nodal is re-expressed in the melanoma xenografts and tumorigenicity resumes. This intriguing finding suggests that targeted and sustained therapy against a key developmental factor, such as Nodal, may represent a new therapeutic approach to treating cancer.

\section{Conclusion}

Clearly, the embryonic microenvironment (such as the one associated with hESCs) is remarkable in its capacity to foster exquisitely complex developmental and regulatory processes in both time and space, ultimately supporting normal cellular differentiation. Here, we demonstrate the epigenetic influence of the hESC microenvironment on the reprogramming of aggressive melanoma cells using an innovative 3-D model. Specifically, our laboratory has previously demonstrated the redifferentiation of these melanoma cells to a more melanocyte-like phenotype [1], and now we show the loss of VE-Cadherin expression (indicative of a plastic vasculogenic phenotype) and the loss of Nodal expression (a plasticity stem cell marker) in tumor cells exposed to the hESC microenvironment. Although quite intriguing, this reprogramming event appears to be transient, as melanoma cells revert back to their original plastic phenotype 1 week after removal from the hESC microenvironment. Further studies with the 3-D culture model revealed the epigenetic influence of aggressive melanoma cells on hESCs resulting in the downregulation of plasticity markers and the emergence of phenotype-specific genes. Additional studies with the aggressive melanoma conditioned matrix microenvironment demonstrated the transdifferentiation of normal melanocytes into melanoma-like cells exhibiting a vasculogenic phenotype. Collectively, these studies have advanced our understanding of the epigenetic influence associated with the microenvironments of hESCs and aggressive melanoma cells, and shed new light on their therapeutic implications. Moreover, we have a better appreciation of the convergence of embryonic and tumorigenic signaling pathways that might stimulate further consideration of targeting Nodal in aggressive tumor cells resulting in a down-regulation of tumorigenic potential and plasticity. Therapeutic strategies will continue to target tumor cells themselves, but we must not neglect the impact that tumor cells have on their surrounding cellular and stromal components. As discovery proceeds, we may recognize that antagonizing tumor/microenvironment signaling is a crucial component of any effective therapy, disallowing aggressive tumor cells to manipulate their surroundings to facilitate their progression. The novel nature of these advances should only improve as our understanding becomes more sophisticated.

Acknowledgments The authors are grateful for the scientific expertise and discussions with our colleagues Drs. Brian Nickoloff at Loyola University, Paul Kulesa and Jennifer Kasemeier-Kulesa at the Stowers Institute for Medical Research, Jolanta Topczewska and Jacek Topczewski at the Children's Memorial Research Center of Northwestern University. This work is supported by NIH grants CA59702, CA121205, an Illinois Regenerative Medicine Institute grant, and a Charlotte Geyer Foundation grant (MJCH).

\section{References}

1. Postovit LM et al (2006) A three-dimensional model to study the epigenetic effects induced by the microenvironment of human embryonic stem cells. Stem Cells 24(3):501-505

2. Hendrix MJ et al (2007) Reprogramming metastatic tumour cells with embryonic microenvironments. Nat Rev Cancer 7(4):246255

3. Seftor EA et al (2002) Expression of multiple molecular phenotypes by aggressive melanoma tumor cells: role in vasculogenic mimicry. Crit Rev Oncol Hematol 44(1):17-27

4. Geng L et al (2007) Hedgehog signaling in the murine melanoma microenvironment. Angiogenesis 10(4):259-267

5. Topczewska JM et al (2006) Embryonic and tumorigenic pathways converge via Nodal signaling: role in melanoma aggressiveness. Nat Med 12(8):925-932 
6. Balint $\mathrm{K}$ et al (2005) Activation of Notch1 signaling is required for beta-catenin-mediated human primary melanoma progression. J Clin Invest 115(11):3166-3176

7. Weeraratna AT et al (2002) Wnt5a signaling directly affects cell motility and invasion of metastatic melanoma. Cancer Cells 1 (3):279-288

8. Stecca B et al (2007) Melanomas require HEDGEHOG-GLI signaling regulated by interactions between GLI1 and the RAS-MEK/AKT pathways. Proc Natl Acad Sci U S A 104(14):5895-5900

9. Liu ZJ et al (2006) Notch1 signaling promotes primary melanoma progression by activating mitogen-activated protein kinase/phosphatidylinositol 3-kinase-Akt pathways and up-regulating Ncadherin expression. Cancer Res 66(8):4182-4190

10. Mintz B, Illmensee K (1975) Normal genetically mosaic mice produced from malignant teratocarcinoma cells. Proc Natl Acad Sci U S A 72(9):3585-3589

11. Illmensee K, Mintz B (1976) Totipotency and normal differentiation of single teratocarcinoma cells cloned by injection into blastocysts. Proc Natl Acad Sci U S A 73(2):549-553

12. Dolberg DS, Bissell MJ (1984) Inability of Rous sarcoma virus to cause sarcomas in the avian embryo. Nature 309(5968):552-556

13. Lee LM et al (2005) The fate of human malignant melanoma cells transplanted into zebrafish embryos: assessment of migration and cell division in the absence of tumor formation. Dev Dyn 233 (4): $1560-1570$

14. Kulesa PM et al (2006) Reprogramming metastatic melanoma cells to assume a neural crest cell-like phenotype in an embryonic microenvironment. Proc Natl Acad Sci U S A 103(10):3752-3757

15. Haldi $M$ et al (2006) Human melanoma cells transplanted into zebrafish proliferate, migrate, produce melanin, form masses and stimulate angiogenesis in zebrafish. Angiogenesis 9(3):139-151

16. Karnoub AE et al (2007) Mesenchymal stem cells within tumour stroma promote breast cancer metastasis. Nature 449(7162):557-563

17. Serafimidis I et al (2007) Novel effectors of directed and ngn3 mediated differentiation of mouse embryonic stem cells into endocrine pancreas progenitors. Stem Cells 26:3-16

18. Radtke F et al (2004) Notch regulation of lymphocyte development and function. Nat Immunol 5(3):247-253

19. Elmi M et al (2007) Mechanism of MASH1 induction by ASK1 and ATRA in adult neural progenitors. Mol Cell Neurosci 36(2):248-259

20. Vallier L, Alexander M, Pedersen RA (2005) Activin/Nodal and FGF pathways cooperate to maintain pluripotency of human embryonic stem cells. J Cell Sci 118(Pt 19):4495-4509

21. Skottman $\mathrm{H}$ et al (2006) Unique gene expression signature by human embryonic stem cells cultured under serum-free conditions correlates with their enhanced and prolonged growth in an undifferentiated stage. Stem Cells 24(1):151-167

22. Constantinescu SN (2000) Stem cell generation and choice of fate: role of cytokines and cellular microenvironment. J Cell Mol Med 4(4):233-248

23. Lensch MW, Daheron L, Schlaeger TM (2006) Pluripotent stem cells and their niches. Stem Cell Rev 2(3):185-201

24. Nemeth MJ et al (2007) Wnt5a inhibits canonical Wnt signaling in hematopoietic stem cells and enhances repopulation. Proc Natl Acad Sci U S A 104(39):15436-15441

25. Babaie $Y$ et al (2007) Analysis of Oct4-dependent transcriptional networks regulating self-renewal and pluripotency in human embryonic stem cells. Stem Cells 25(2):500-510
26. Ogawa K et al (2007) Activin-Nodal signaling is involved in propagation of mouse embryonic stem cells. J Cell Sci 120(Pt 1): $55-65$

27. Balasubramaniyan V et al (2006) Effects of histone deacetylation inhibition on neuronal differentiation of embryonic mouse neural stem cells. Neuroscience 143(4):939-951

28. Londin ER, Mentzer L, Sirotkin HI (2007) Churchill regulates cell movement and mesoderm specification by repressing Nodal signaling. BMC Dev Biol 7(1):120

29. Mita K, Fujiwara S (2007) Nodal regulates neural tube formation in the Ciona intestinalis embryo. Dev Genes Evol 217(8):593-601

30. Tabibzadeh S, Hemmati-Brivanlou A (2006) Lefty at the crossroads of "stemness" and differentiative events. Stem Cells 24 (9):1998-2006

31. Branford WW, Yost HJ (2002) Lefty-dependent inhibition of Nodal- and Wnt-responsive organizer gene expression is essential for normal gastrulation. Curr Biol 12(24):2136-2141

32. Hendrix MJ et al (2001) Expression and functional significance of VE-cadherin in aggressive human melanoma cells: role in vasculogenic mimicry. Proc Natl Acad Sci U S A 98(14):80188023

33. Hess AR et al (2006) VE-cadherin regulates EphA2 in aggressive melanoma cells through a novel signaling pathway: implications for vasculogenic mimicry. Cancer Biol Ther 5(2):228-233

34. Postovit LM et al (2007) The commonality of plasticity underlying multipotent tumor cells and embryonic stem cells. J Cell Biochem 101(4):908-917

35. Bissell MJ, Radisky D (2001) Putting tumours in context. Nat Rev Cancer 1(1):46-54

36. Seftor RE et al (2001) Cooperative interactions of laminin 5 gamma2 chain, matrix metalloproteinase-2, and membrane type-1matrix/metalloproteinase are required for mimicry of embryonic vasculogenesis by aggressive melanoma. Cancer Res 61 (17):6322-6327

37. Kalluri R (2003) Basement membranes: structure, assembly and role in tumour angiogenesis. Nat Rev Cancer 3(6):422-433

38. Kenny PA, Lee GY, Bissell MJ (2007) Targeting the tumor microenvironment. Front Biosci 12:3468-3474

39. Seftor RE et al (2002) Targeting the tumor microenvironment with chemically modified tetracyclines: inhibition of laminin 5 gamma2 chain promigratory fragments and vasculogenic mimicry. Mol Cancer Ther 1(13): 1173-1179

40. Seftor EA et al (2005) Epigenetic transdifferentiation of normal melanocytes by a metastatic melanoma microenvironment. Cancer Res 65(22):10164-10169

41. Valdimarsdottir G, Mummery C (2005) Functions of the TGFbeta superfamily in human embryonic stem cells. APMIS 113(11-12): 773-789

42. Brennan J, Norris DP, Robertson EJ (2002) Nodal activity in the node governs left-right asymmetry. Genes Dev 16(18):2339-2344

43. Postovit LM et al (2007) Targeting Nodal in malignant melanoma cells. Expert Opin Ther Targets 11(4):497-505

44. Schier AF (2003) Nodal signaling in vertebrate development. Annu Rev Cell Dev Biol 19:589-621

45. Chen C, Shen MM (2004) Two modes by which Lefty proteins inhibit nodal signaling. Curr Biol 14(7):618-624

46. Cheng SK et al (2004) Lefty blocks a subset of TGFbeta signals by antagonizing EGF-CFC coreceptors. PLoS Biol 2(2):E30 